“C 2018 IEEE. Personal use of this material is permitted. Permission from IEEE must be obtained for all other uses, in any current or future media, including reprinting/republishing this material for advertising or promotional purposes, creating new collective works, for resale or redistribution to servers or lists, or reuse of any copyrighted component of this work in other works." 


\section{PDMS-Embedded Conductive Fabric: A Simple Solution for Fabricating PDMS-Based Wearable Antennas with Robust Performance}

\author{
Roy B. V. B. Simorangkir, Shilun Feng, \\ Abu Sadat Md. Sayem, Karu P. Esselle \\ School of Engineering \\ Macquarie University \\ Sydney, NSW 2109, Australia \\ Email: roy.basten@students.mq.edu.au
}

\author{
Yang Yang \\ School of Electrical and Data Engineering \\ University of Technology Sydney \\ Ultimo, Sydney, NSW 2007, Australia \\ Email: yang.yang.au@ieee.org
}

\begin{abstract}
A new and simple fabrication method to realize robust flexible wearable antennas by combining conductive fabric and polydimethylsiloxane (PDMS) is proposed. The conductive fabric acts as the conductive part of the antenna, while PDMS acts as the substrate as well as the encapsulation layers. The method takes advantage of the porous structure of the conductive fabric and the initial liquid form of PDMS to attain a significantly strong integration between the two, leading to a robust PDMSbased wearable antenna. A number of patch antennas have been designed, fabricated, and tested to validate the proposed concept and the results are presented, showing robust performance. The fabricated prototypes have a resonance frequency approximately at $2.46 \mathrm{GHz}$ with a $10 \mathrm{~dB}$ return-loss bandwidth ranging from 3.3 to $5.7 \%$.
\end{abstract}

\section{INTRODUCTION}

Polydimethylsiloxane (PDMS) has been considered one of the most promising materials for the realization of wearable antennas. The unique characteristics of PDMS, including extreme flexibility [1], acceptable loss and tunable relative permittivity [2], water resistance, transparency, temperature stability [3], and simple preparation [4], make it advantageous over its counterparts, such as textiles, paper, or any other polymers.

An issue in flexible antenna development using PDMS is, however, the inherent incompatibility of PDMS and metal. To achieve a strong adhesion between the PDMS and the antenna conductive parts which can stand extreme deformation and stress is therefore a challenging process. There have been a number of successful solutions to this issue, including substrate pretreatment through oxygen plasmas exposure [5], embedding carbon nanotube sheets [4], injecting liquid metal [6], and embedding silver nanowires (AgNWs) [7]. However, in general these approaches require a quite complex fabrication process.

In this paper, we present a simple, yet effective approach to overcome the aforementioned problems, allowing for a relatively easy realization of flexible and mechanically robust PDMS-based wearable antennas. In the following text, the proposed method is described. As concept demonstrations, four inset-fed patch antennas have been fabricated and tested, validating the applicability of the proposed approach.

\section{Proposed Method}

The proposed solution is to utilize conductive fabric as the conductive parts of the antenna which then are embedded inside PDMS layers. Different to our work in [8], where the conductive fabric is simply adhered to the PDMS surface, this approach provides a stronger structural integration between the conductive and nonconductive parts of the antenna. That is due to the support from the PDMS-PDMS layers adhesion created through the pores of the fabric, which is much stronger than that of the PDMS and conductive fabric. As a result, this approach allows for a realization of PDMS-based antennas with a robust performance against very harsh operating conditions.

\section{MATERIALS FOR REALIZATION}

For the antenna conductive parts, i.e. the ground plane and patch, woven conductive fabrics are considered for their high and isotropic effective conductivity, compared to those of knitted conductive fabrics [9]. Multifilament entirely plated threads intertwine with each other in a one-to-one ratio between the vertical and horizontal directions, and the distance between the adjacent threads is very small, less than $0.04 \mathrm{~mm}$, thus allowing a good resemblance to a solid metallic plate [10]. There are four different conductive fabrics from Marktek Inc. and Less EMF Inc. considered in this work, named CF I, II, III, and IV, respectively. The major differences between these fabrics are their thicknesses, the composition of metal coating on the threads, and the threads density, which lead to different effective conductivities, hence affecting the performance of the fabricated antenna.

To fabricate the PDMS layers, a Dow Corning Sylgard 184 silicone elastomer kit was employed. Firstly, the base and the curing agent of the kit were mixed at room temperature with a ratio of 10 to 1 , followed by degassing the mixed solution in a vacuum desiccator to remove bubbles possibly formed during the mixing. Afterwards, the bubble-free solution 


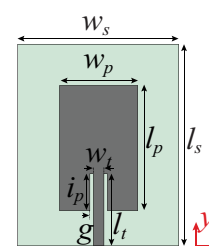

Patch layer

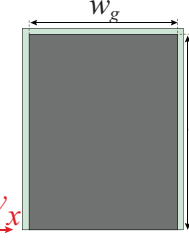

Ground layer

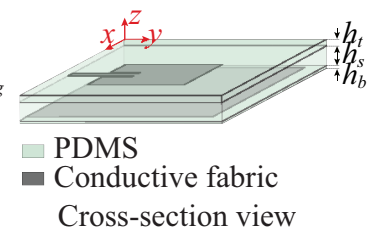

Cross-section view

Fig. 1. Antennas designed for concept demonstration.

TABLE I

Detailed Dimensions of Patch Antennas

\begin{tabular}{c|c|c|c|c}
\hline \multirow{2}{*}{ Parameter } & \multicolumn{4}{|c}{ Antenna Dimension $(\mathrm{mm})$} \\
\cline { 2 - 5 } & CF I & CF II & CF III & CF IV \\
\hline \hline$w_{s}$ & 50.3 & 50.3 & 50.3 & 50.3 \\
$l_{s}$ & 59.6 & 59.5 & 59.5 & 58.7 \\
$w_{g}$ & 42.3 & 42.3 & 42.3 & 42.3 \\
$l_{g}$ & 55.6 & 55.5 & 55.5 & 54.7 \\
$w_{p}$ & 22.3 & 22.3 & 22.3 & 22.3 \\
$l_{p}$ & 35.6 & 35.5 & 35.5 & 34.7 \\
$w_{t}$ & 3 & 3 & 3 & 3 \\
$l_{t}$ & 21 & 19 & 19 & 13.5 \\
$i_{p}$ & 11 & 9 & 9 & 3.5 \\
$g$ & 1 & 1 & 1 & 1 \\
$h_{t}$ & 0.76 & 0.76 & 0.76 & 0.76 \\
$h_{s}$ & 2.5 & 2.5 & 2.5 & 2.5 \\
$h_{b}$ & 0.2 & 0.2 & 0.2 & 0.2 \\
\hline
\end{tabular}

was tape-casted or poured into the target mold, to ensure the desired thickness of the PDMS layer. If necessary, the degassing process can be repeated to ensure that no air bubbles are left inside the solution. The last step was the curing of the solution that can be done at room temperature for $24 \mathrm{~h}$ or under an elevated temperature $\left(65^{\circ} \mathrm{C}\right.$ for $\left.2 \mathrm{~h}\right)$ to expedite the polymerization.

\section{Concept Demonstrations}

\section{A. Antenna Configuration}

To validate the applicability of the proposed approach for realization of flexible antennas, the combinations of PDMS and four conductive fabrics were applied to an inset-fed rectangular patch antenna design illustrated in Fig. 1. PDMS layers are used as the substrate and encapsulation that covers completely the antenna. For each combination, the antenna was optimized to have a fundamental-mode operation at ISM $2.45 \mathrm{GHz}$. Table I gives the dimensions of the final designs which were then fabricated through the process in [11], [12]. Four fabricated prototypes are shown in Fig. 2.

\section{B. Measurements and Results}

The RF performance of all prototypes were characterized, including input reflection coefficient $\left(\left|S_{11}\right|\right)$ measurements using the Agilent PNA-X N5242A network analyzer, calibrated with an electronic calibration module N4691B from Keysight,

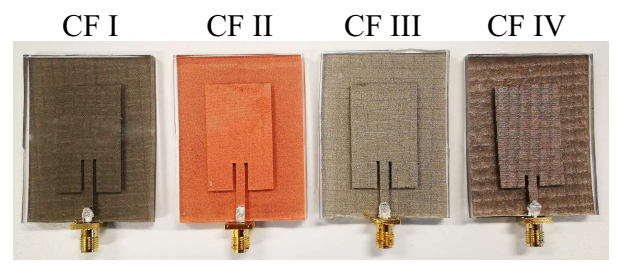

Fig. 2. Fabricated patch antennas.

TABLE II

Summary of the PeAK Gains AND EFFiciencies of the Prototypes IN FREE SPACE

\begin{tabular}{c|c|c|c|c}
\hline \multirow{2}{*}{ Antenna } & \multicolumn{2}{|c|}{ Gain $(\mathrm{dBi})$} & \multicolumn{2}{c}{ Eff.(\%) } \\
\cline { 2 - 5 } & Sim. & Meas. & Sim. & Meas. \\
\hline \hline CF I & 1.85 & 1.75 & 36.3 & 35.6 \\
CF II & 1.47 & 1.36 & 33.1 & 31.5 \\
CF III & 1.53 & 1.45 & 33.6 & 32.1 \\
CF IV & -0.74 & -0.82 & 20.1 & 19.7 \\
\hline
\end{tabular}

and far-field measurements in the NSI700S-50 spherical nearfield antenna range at the Australian Antenna Measurement Facility (AusAMF), Marsfield. As shown in Figs. 3(a)-(d), there is a good agreement between the measured $\left|S_{11}\right|$ results and those of the simulations. The fabricated prototypes have a resonance approximately at $2.46 \mathrm{GHz}$ with a 10 return-loss bandwidth ranging from 3.3 to $5.7 \%$. The minor differences between the simulated and measured results might be caused by fabrication error during the manual cutting of the fabric, especially CF IV whose edges easily suffered from fraying. When the antennas were bent around a plastic tube having a radius of $35 \mathrm{~mm}$ (see Fig. 4), the antennas remained functional despite slight shifts in their resonance frequencies, especially during bending along the $E$-plane of the antenna (see Figs. 3(a)-(d)). This is expected as it affects the main current path of the antenna. However, most importantly the conductive fabric remains intact inside the PDMS encapsulation. The desired boresight radiations are achieved from the far-field measurements as shown in simulations (see Figs. 5(a)-(d)). Further, the gains and efficiencies of all prototypes, measured at their resonance frequencies, are given in Table II, and they again agree well with the simulated results.

\section{CONCLUSion}

A simple yet effective approach for realizing flexible and mechanically robust wearable antennas has been presented. The proposed approach was validated by studying the performance of fabricated rectangular patch antennas. A good agreement is shown between the simulated and measured results, validating the proposed method.

\section{REFERENCES}

[1] I. D. Johnston, D. K. McCluskey, C. K. L. Tan, and M. C. Tracey, "Mechanical characterization of bulk Sylgard 184 for microfluidics and microengineering," J. Micromechanics and Microengineering, vol. 24, no. 3, p. 035017, 2014. 


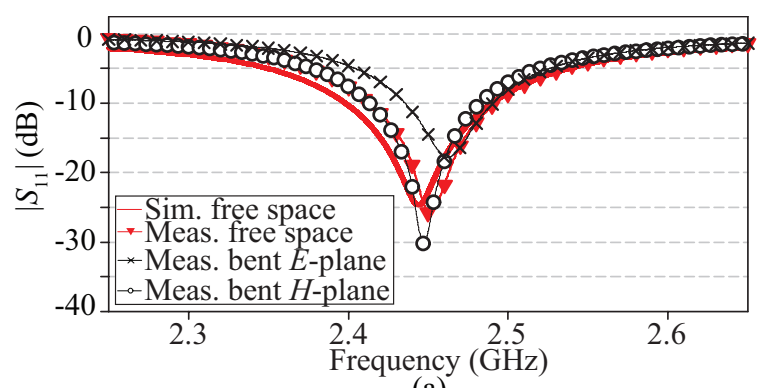

(a)

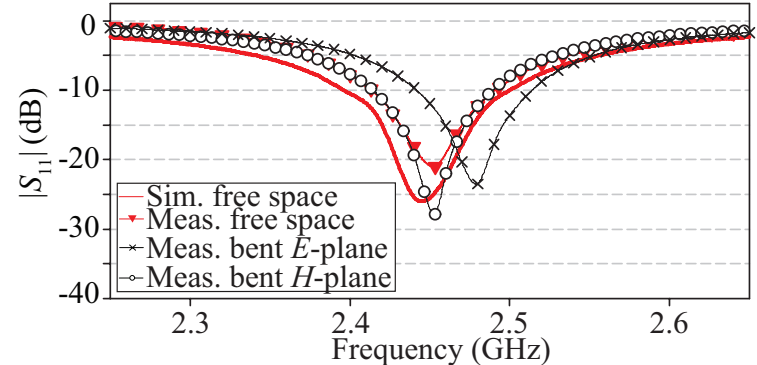

(b)

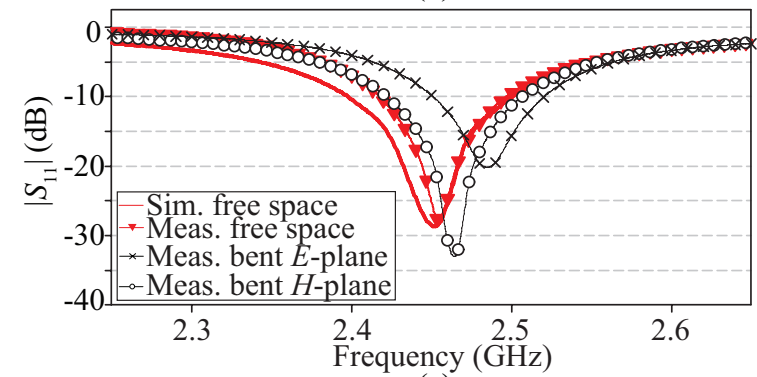

(c)

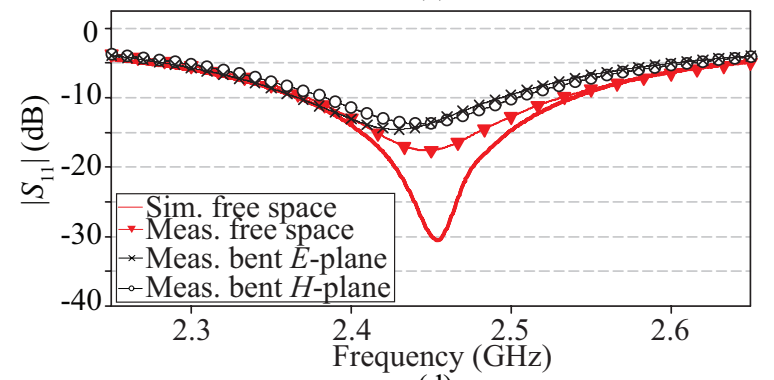

(d)

Fig. 3. Simulated and measured $\left|S_{11}\right|$ of the prototype with: (a) CF I, (b) CF II, (c) CF III, and (d) CF IV.

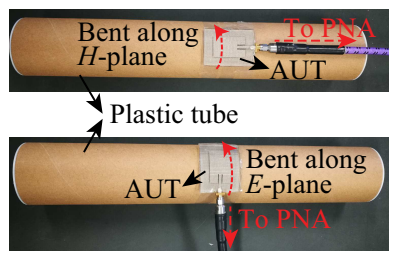

Fig. 4. Illustration of the antenna bending around the plastic tube.

[2] S. Koulouridis, G. Kiziltas, Y. Zhou, D. J. Hansford, and J. L. Volakis, "Polymer-ceramic composites for microwave applications: Fabrication and performance assessment," IEEE Trans. Microw. Theory Tech., vol. 54, no. 12, pp. 4202-4208, Dec 2006.

[3] A. R. Colas, "Silicones: preparation, properties and performances," Chimie Nouvelle, The Journal of the Societe Royale de Chimie, pp.
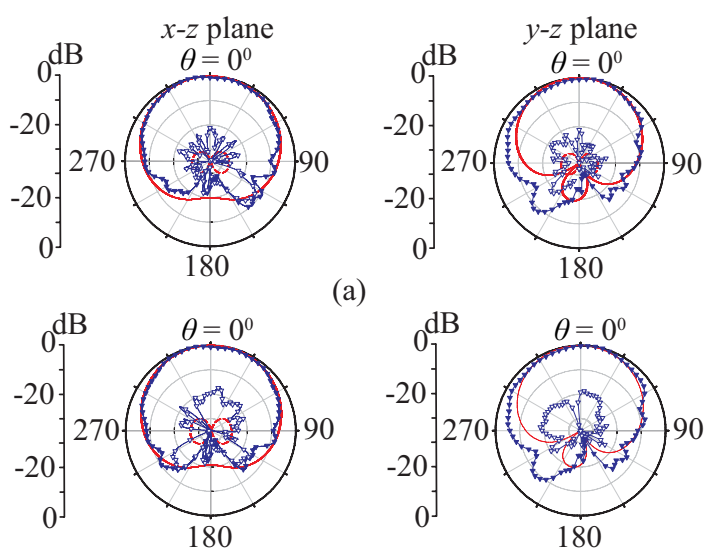

(b)
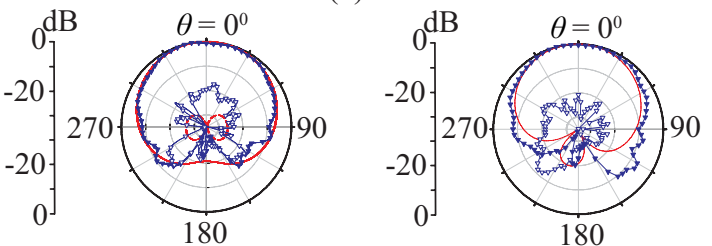

(c)
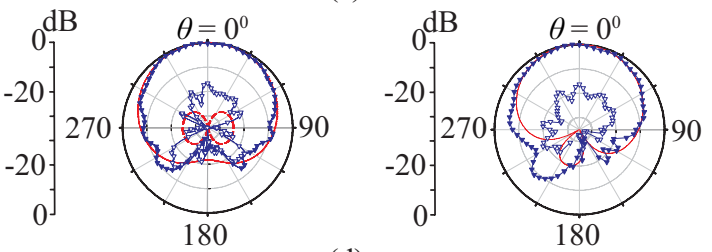

(d)

$\begin{array}{ll}- \text { Predicted Co-pol } & \rightarrow \text { - Measured Co-pol } \\ --- \text { Predicted X-pol } & \rightarrow \text { Measured X-pol }\end{array}$

Fig. 5. Normalized simulated and measured radiation patterns of the prototype with: (a) CF I, (b) CF II, (c) CF III, and (d) CF IV.

847-852, 1990.

[4] Y. Zhou, Y. Bayram, F. Du, L. Dai, and J. L. Volakis, "Polymer-carbon nanotube sheets for conformal load bearing antennas," IEEE Trans. Antennas Propag., vol. 58, no. 7, pp. 2169-2175, July 2010.

[5] P. Bodo and J.-E. Sundgren, "Titanium deposition onto ion-bombarded and plasma-treated polydimethylsiloxane: Surface modification, interface and adhesion," Thin Solid Films, vol. 136, no. 1, pp. 147-159, 1986.

[6] G. J. Hayes, J. H. So, A. Qusba, M. D. Dickey, and G. Lazzi, "Flexible liquid metal alloy (EGaIn) microstrip patch antenna," IEEE Trans. Antennas Propag., vol. 60, no. 5, pp. 2151-2156, May 2012.

[7] G.-W. Huang, H.-M. Xiao, and S.-Y. Fu, "Wearable electronics of silvernanowire/poly(dimethylsiloxane) nanocomposite for smart clothing." Scientific Reports, vol. 5, no. 2, p. 13971, 2015.

[8] R. B. V. B. Simorangkir, Y. Yang, L. Matekovits, and K. P. Esselle, "Dual-band dual-mode textile antenna on PDMS substrate for bodycentric communications," IEEE Antennas Wireless Propag. Lett., vol. 16 , pp. 677-680, 2017.

[9] Y. Ouyang and W. J. Chappell, "High frequency properties of electrotextiles for wearable antenna applications," IEEE Trans. Antennas Propag., vol. 56, no. 2, pp. 381-389, Feb 2008.

[10] B. Ivsic, D. Bonefacic, and J. Bartolic, "Considerations on embroidered textile antennas for wearable applications," IEEE Antennas Wireless Propag. Lett., vol. 12, pp. 1708-1711, 2013.

[11] R. B. V. B. Simorangkir, Y. Yang, K. P. Esselle, and Y. Diao, "A varactortuned frequency-reconfigurable fabric antenna embedded in polymer: Assessment of suitability for wearable applications," in 2017 IEEE MTT S International Microwave Symposium (IMS), June 2017, pp. 204-207.

[12] R. B. V. B. Simorangkir, Y. Yang, K. P. Esselle, and B. A. Zeb, "A method to realize robust flexible electronically tunable antennas using polymer-embedded conductive fabric," IEEE Trans. Antennas Propag., vol. 66, no. 1, pp. 50-58, Jan 2018. 